\title{
Evaluation of Vaccination Strategy Against Rabies in Hong Kong Macaques
}

\author{
Omid Nekouei $^{1}$, Paolo Martelli ${ }^{2}$, Sophie St-Hilaire ${ }^{1}$, Hui Suk Wai ${ }^{2}$, Karthiyani \\ Krishnasamy $^{2}$, and Ioannis Magouras ${ }^{1}$ \\ ${ }^{1}$ City University of Hong Kong \\ ${ }^{2}$ Ocean Park Conservation Foundation Hong Kong
}

November 22, 2021

\begin{abstract}
Rabies is a fatal zoonotic disease that can affect all mammals. Following the directives of the rabies ordinance of the Government of Hong Kong, all wild macaques captured under an ongoing sterilization program (since 2000) were vaccinated against rabies. The main objective of this study was to assess the serological response to rabies vaccination in the population of Hong Kong macaques. An inactivated rabies vaccine was subcutaneously administered to captured macaques under anesthesia. In a 2015 field survey, blood samples from the animals were collected and stored in - 80 freezer. In July 2021, all frozen sera from vaccinated animals were prepared and tested for antibodies against rabies virus using a commercial enzyme-linked immunosorbent assay (ELISA) test. The test results were dichotomized at the recommended cut-off point of the test kit. Sixty-five samples from the vaccinated macaques were available for this study. All of these animals had received at least one dose of vaccine (1 st vaccination) between 2008 and 2015. The interval between the $1^{\text {st }}$ vaccination and blood sampling dates ranged from 21 to 2,779 days. Only five of the 65 macaques had a second vaccination record at the time of sampling; all five had high antibody levels. Among the remaining macaques, $77 \%$ (46/60) were positive for rabies antibodies. No specific association was observed between the post-vaccination period and the antibody titer of these macaques and no adverse reactions to vaccination were reported. The current vaccination strategy in Hong Kong macaques appears to effectively elicit rabies antibodies in a high proportion of macaque populations in the wild (78-87\%). However, reaching the precise level of protection against a potential challenge with the virus should further be investigated.
\end{abstract}

\section{Hosted file}

Full Manuscript File_TED.docx available at https://authorea.com/users/447389/articles/546392evaluation-of-vaccination-strategy-against-rabies-in-hong-kong-macaques 